\title{
Discursos sobre normas relativas a sexualidad en jóvenes del norte de Chile
}

Discourses on rules related to sexuality in youths in northern Chile

\author{
Jimena Silva-Segovia / jsilva@ucn.cl \\ Universidad Católica del Norte, Chile \\ Leyla Méndez-Caro / leyla.mendez@uantof.cl \\ Universidad de Antofagasta, Chile \\ Jaime Barrientos-Delgado/ jbarien@ucn.cl \\ Universidad Católica del Norte, Chile
}

\begin{abstract}
This study aimed at learning institutional discourses on the sexuality and selfcare youngsters. Participants were between 15 and 19 years of age, men and women from public, private, and university educational institutions from the city of Antofagasta in northern Chile. A qualitative methodology was used and 24 subjects were interviewed in-depth. The main findings suggest a flexible relation of young subjects with institutional regulations, either ascribing to their mandates or exercising dissent. Poor implementation of sexual education programs was detected, thus becoming a problem throughout private and public institutions, although with greater social impact on the latter.
\end{abstract}

Key words: youths, discourses, normative institutions, sexualities.

Resumen: Esta investigación tuvo por objetivo conocer los discursos institucionales de la sexualidad y el autocuidado juvenil. Los participantes fueron hombres y mujeres jóvenes de entre 15 y 19 años, pertenecientes a establecimientos educativos públicos, privados y universitarios de la ciudad de Antofagasta, en el norte de Chile. Se trabajó con una metodología cualitativa, aplicando entrevistas en profundidad a 24 sujetos. Los principales hallazgos sugieren una relación flexible del sujeto joven con las normativas institucionales, en algunos casos adscribiéndose a sus mandatos y, en otros, ejerciendo disidencias. Asimismo, se advirtió una implementación deficiente de programas en educación sexual, constituyendo una problemática transversal en establecimientos privados y públicos, aunque con mayores repercusiones sociales en estos últimos.

Palabras clave: juventudes, discursos, instituciones normativas, sexualidades. 


\section{Introducción $^{1}$}

En este trabajo buscamos comprender: ¿ cómo operan los discursos valóricos provenientes de instituciones como la Iglesia, el Estado o la escuela sobre la sexualidad juvenil de estudiantes antofagastinos/as? y ¿cómo estos/as jóvenes se relacionan con los discursos institucionales que recaen sobre el cuidado de sus cuerpos? Elegimos analizar los discursos de tres instituciones normativas: Iglesia, escuela y Estado, por constituir en Latinoamérica núcleos históricos de poder discursivo en la formación valórica y de subjetividades en la sociedad (Vera, 2005).

Dichos núcleos nos permiten reflexionar sobre la trama sociocultural contemporánea, sin olvidar las raíces de creencias y prácticas que han marcado las estructuras normativas que regulan los comportamientos sexuales. Asimismo son instituciones que sostienen los ideales culturales del ser joven y el ejercicio moral de sujeto en la sociedad. Estas tres instituciones si bien operan de modos aparentemente independiente, en Latinoamérica se articulan y retroalimentan entre sí, en las grandes decisiones políticas.

En el contexto latinoamericano, observamos la emergencia de una ciudadanía juvenil alternativa, que emerge con vigor desde los años $2000 \mathrm{a} \mathrm{diez}$ años posdictaduras, donde "toma conciencia de su pertenencia en la sociedad y el mundo de su tiempo [renunciando] a una posición de simple espectador [para] colocar su pensamiento o su arte al servicio de una causa" (Borda, 1981: 56). Los jóvenes quiebran la metáfora de generación improductiva y aceleran una crisis de representatividad de las políticas dominantes.

Esta generación juvenil construye espacios de mayor agudización social y contradicciones, adoptando posiciones de interpelación al sistema capitalista imperante en el mundo occidental y que ha permeado las sociedades latinoamericanas. La condición juvenil latinoamericana se supedita a la condición de clase, pues ésta la supera y le es anterior (Novaes y Santoro, 2008). Así, los grupos populares de acción juvenil han sido protagonistas de los grandes cambios actuales posdictaduras.

Durante los años noventa en Chile, predominó el legado de la dictadura militar (1973-1990), periodo durante el cual se exacerbó el accionar de diferentes instituciones, especialmente del Estado y la Iglesia, así como de la familia y la escuela, como dispositivos de formación discursiva basados en

1 Esta investigación ha recibido el apoyo de Fondecyt 1110301 y la colaboración de tesistas de Psicología de la Universidad Católica del Norte, en la producción de entrevistas y grupos de conversación. 
la normalización y el control de la sexualidad. La familia que es considerada símbolo de reconciliación nacional y receptáculo de esperanzas de la cohesión social (Grau, 1997; Vera, 2009) es permeado por la moral católica, institución que juega un rol fundamental como jueza y guardiana de los valores nacionales (Htun, 2010; Urriola, 2008). En este escenario, Chile era hasta hace poco el único país en el mundo, además de Malta, donde el divorcio era ilegal. Asimismo, Chile está entre el pequeño grupo de países donde el aborto es ilegal en todas las circunstancias, incluso para salvar la vida de la madre (Htun, 2010).

Si bien estas instituciones han marcado la construcción de subjetividades durante toda una época, varios autores (Barrientos, 2006; Htun, 2010; Ruiz et al., 2011) advierten que, a medida que avanza el periodo posdictatorial, se observa una desamarre de estas instituciones tradicionales (Iglesia, familia, Estado y escuela), a través del cual se flexibilizan las formas de vida y cada vez más "las reglas [van] dejando de ser imperativos externos, absolutos e intangibles fundados en criterios derivados de la religión o un grupo social; cada vez menos, valen para vigilar o fustigar la juventud" (Barrientos, 2006: 92).

De esta forma, algunos estudios refieren que los jóvenes adoptan diferentes vectores o posiciones de enunciación frente a las normas (Portocarrero, 2009), en tanto proceso que apunta ya no a "la distancia de los individuos con la norma sino a la lógica de su relación con ella" (Araujo, 2009b: 95). En este sentido, investigaciones en Chile sobre sexualidad y autocuidado nos advierten de esta dinámica, enfatizando en procesos de transformación social, donde se fortalecen espacios de acción potencial de los sujetos, haciendo posible mayor reflexibilidad y distancia crítica hacia las normas (Guzmán y Godoy, 2009).

De esta forma, las mujeres de generaciones jóvenes reconocen cada vez más el amor, el deseo y la curiosidad (Palma, 2007), y los jóvenes de sectores socioeconómicos medios y altos están postergando la formación de parejas y maternidad; mientras que en sectores populares se incrementa el número de embarazos juveniles (57\%) (Palma, 2010), disminuyendo este porcentaje a medida que aumenta el nivel socioeconómico (INJUv, 2009; Gallegos et al., 2009). De igual modo, en este grupo la maternidad se resignifica como un proceso de búsqueda de identidad y legitimidad social, constituyéndose en un factor de reproducción de la pobreza intergeneracional (Guzmán y Godoy, 2009).

Estas voces disidentes, que se contraponen a ideologías dominantes, han incorporado nuevos modos de vivir en sociedad, en tanto transformación de 
pautas socioculturales que la regulan, modificando sus funciones y efectos e invitando a reflexionar sobre una nueva normatividad en el comportamiento sexual juvenil (Barrientos, 2006; Bozon, 2004).

El contexto sociocultural donde los/as jóvenes participantes construyen sus normas es de predominancia minera derivada de su principal actividad productiva regional, de allí la necesidad de indagar en cómo un contexto altamente conservador en torno a las relaciones de género impacta en la subjetividad y en la construcción de normas y valores. Algunos estudios refieren que a las mujeres se les dificulta incorporar en sus significaciones una sexualidad pensada como un derecho que les permita encarar el sexismo imperante y así poder enfrentar múltiples formas de violencia inter e intra género (Olavarría, 2009). Los hombres reafirman el ejercicio de la sexualidad como un privilegio masculino, lo cual otorga prestigio social entre pares y frente a hombres mayores (Salinas y Barrientos, 2011).

\section{Método}

Esta investigación se desarrolla bajo un modelo cualitativo, con enfoque de género, donde la captura de los procesos biográficos de construcción y deconstrucción de las experiencias de los jóvenes se realiza a través de entrevistas. Dicha técnica permitió contrastar los procesos intersubjetivos de comunicación individual entre investigadores y participantes, lo cual facilitó la emergencia de discursos ideológicos y las representaciones simbólicas en sus mundos sociales (Alonso, 2003; Bourdieu, 2009). Se incluyeron 24 hombres y mujeres jóvenes de Antofagasta, Chile ( 4 hombres y 4 mujeres de colegios privados, 2 hombres y 5 mujeres de colegios públicos, y 6 hombres y 3 mujeres de universidades). Los criterios de inclusión fueron: edad 15-19 años y pertenecer a un establecimiento de educación. La selección de participantes se llevó a cabo mediante un muestreo teórico intencional, bajo el cual se incluyó nuevos participantes en la medida que fueran aportando nuevos conceptos al análisis, buscando así saturación en sus discursos (Méndez y Cárdenas, 2012).

La producción de relatos individuales se realizó mediante un guión semiestructurado en las siguientes dimensiones: a) Instituciones normativas, castigos, restricciones y controles, $b$ ) instituciones normativas y socialización de género, $c$ ) creencias religiosas en la vivencia de la sexualidad y gestión de riesgos y autocuidado, $d$ ) educación sexual en Chile.

Para salvaguardar los criterios éticos, se solicitó la firma de asentimientos, consentimientos y autorizaciones, que fueron suscritos por los padres 
de los participantes; esto se coordinó con cada establecimiento educativo. También cada estudiante firmó un asentimiento. Se asignó un código a cada entrevista para garantizar el anonimato.

En el proceso interpretativo hemos seguido algunos lineamientos del análisis de discurso y análisis narrativo, que han servido de enseñanzas para organizar el material (De-Villers, 1999; Sharim, 2005; Van Dijk, 2008). Hemos hecho hincapié en el tratamiento de las producciones simbólicas, a partir de los aportes de estos autores, que nos permiten comprender, analizar e interpretar las condiciones sociales de producción, y la posición de los sujetos en el ámbito social que figura en el cuerpo de las narrativas (Bourdieu, 2008). Destacamos la producción de significados y la importancia de las experiencias de los jóvenes inscritos en los diferentes centros educativos. Se llevaron a cabo contrapuntos teóricos seleccionados con todo el material.

Este proceso implicó cuatro fases: 1) descubrimiento en progreso: identificación de ejes temáticos y categorías; 2) selección de microtextos, edición de relatos, codificación y debates teóricos en equipo; 3) construcción de grillas para el reordenamiento de los microtextos y su análisis interpretativo; 4) interpretación y contrapunto teórico, que permite dar cuenta de la aproximación disciplinaria. En ese sentido, hemos elegido un abordaje interdisciplinario con base en la psicología (Sharim, 2005; Barrientos, 2006; Araujo, 2009a; Vera, 2009; Palma, 2010) articulando con la sociología (Dides, 2007; Bozón, 2004; Borda, 1981; Foucault, 2002; Bourdieu, 2008) y antropología (Silva, 2009; Marín, 2008), transversalizando con la Teoría de género. Estas elecciones nos han permitido situar la subjetividad juvenil, en una dialéctica que analiza las tensiones entre las instituciones y sus mundos sociosimbólicos vinculados con la sexualidad.

Los criterios de validación utilizados fueron la triangulación de actores (hombres, mujeres) y triangulación de expertos (investigadores) (Alonso, 2003).

\section{Hallazgos}

A partir del análisis de las entrevistas emergieron los siguientes hallazgos, los cuales nos ayudaron a responder cómo los sujetos jóvenes se relacionan con los discursos institucionales provenientes de la Iglesia, el Estado y la escuela en la vivencia de la sexualidad y el cuidado de sus cuerpos. 
Iglesia

En esta primera categoría, observamos en el discurso de los jóvenes oscilaciones entre obediencias, disidencias e interpelaciones frente a discursos institucionales provenientes de la Iglesia católica. Esta dinámica, si bien se desarrolla en las tres instituciones analizadas, es mucho más visible en esta institución, pues de acuerdo con los relatos de los participantes, ésta es la entidad que ellos reconocen con mayor facilidad, dada su fuerte presencia simbólica en los procesos de socialización. Asimismo, la cercanía a esta institución, a la vez que promueve obediencias, también promueve reflexiones críticas en torno a las prácticas de ésta.

Si bien advertimos debilitamiento en las adscripciones a las normativas de la Iglesia católica, diluyéndose en los participantes procesos de obediencias, éstos se reactivan al momento de discutir temas valóricos controversiales en la sociedad chilena como la homosexualidad y el matrimonio homosexual, el embarazo en jóvenes y el aborto. Ante estos temas, se defienden mandatos clásicos de la Iglesia, aunque en algunos casos, maquillados desde la "tolerancia" y modernización paulatina de éstos, principalmente en colegios privados católicos.

(...) Igual mi mamá siempre se enoja con mi papá por eso, porque yo siempre he sido así como "ah, que asco, dos hombres dándose un beso", si está en la tele digo "ah no, cámbiala” (...) En el X [Colegio Católico] por ejemplo, no se admiten los gay (...) le gritamos, entre más vaca "seai" más hombre eres (hombre, 16 años, colegio privado).

Si yo sé que voy a tener un hijo y estoy de acuerdo pero sé que en el ambiente donde se va a desarrollar mi bebé es malo, hay drogadicción, hay violaciones, es un mal ambiente... yo prefiero mil veces darlo en adopción aunque me duela, pero yo sé que va a ser lo mejor (...) Adopción, pero no matarlo, no aborto (mujer, 17 años, comunidad Vida Cristiana).

En relación con los grupos de análisis y su posición frente a estos temas, observamos dificultades por parte de los estudiantes de establecimientos municipales, para configurar una posición frente a éstos, basada en el campo discursivo de oposiciones u obediencias, advertidas en otros grupos. Los jóvenes sugieren que no manejan información suficiente para poder opinar. Asimismo, en el corpus de sus relatos, dichos temas casi no surgieron de forma espontánea, debiendo ser direccionados, en la mayoría de los casos, a través de preguntas específicas en la pauta de entrevista.

Es que en realidad no sé mucho de esa píldora [del día después], sé que es anticonceptiva (...) sobre el aborto, estoy como más metido en eso porque si en vez de abortar por qué no esperan los nueve meses y lo dan en adopción, si no quieren tener al hijo... o... a 
ver estoy como pensando (...) Según la iglesia que es una abominación para la vida (...) Más bien es decisión personal, yo no sabría decirle a alguien (...) yo no tengo mucha información sobre eso (hombre, 17 años, establecimiento educacional municipal).

Si bien el estudiante busca parámetros normativos desde los cuales regir y estructurar sus discursos, le es difícil encontrarlos, configurando una relación de tensión con las normas hegemónicas frente al aborto y la anticoncepción de emergencia. Sus precarios espacios de experiencia social respecto a la formación discursiva frente a estos temas lo hacen adscribirse superficialmente a ésta, y a la vez esquivarla, permeando procesos de sujeción bajo lo que él denomina "desinformación".

De forma implícita, configura fugas frente a la normativa opresora, a la vez que le es difícil reconocer su posición en la estructura social y los procesos de control normativo. Así también esta situación podría advertirnos de brechas socioeconómicas en la educación y los pocos espacios de reflexión crítica propiciados en contextos de educación pública, a diferencia de sus pares de colegios privados y universitarios.

En otro nivel, emergen discursos en los cuales se reconoce la influencia e intervención normativa de la Iglesia en la sociedad, se cuestionan sus mandatos, pero a la vez se respeta su influencia e, incluso, se denota miedo a su poder y control, en una tensión interesante entre resistencia y aceptación.

(...) obviamente la iglesia, aunque los jóvenes y mucha gente no la "pesque", va a influenciar mucho a la sociedad, porque como ahí salía hablando el obispo (...) si un obispo dice eso, es porque ya tiene una repercusión en la sociedad. Los políticos van a pescar más lo que dice un obispo a lo que dice un joven (...) Yo creo que la iglesia tiene, por lo menos en estos temas, una importancia súper influyente y a la vez negativa para mí... porque (...) es algo castigador: "es malo iniciarse antes [sexualmente] u ocupar anticonceptivos", y lo único que dicen es la abstinencia (...) (mujer, 17 años, universidad).

(...) como que ha perdido mucha influencia [la iglesia]. Como que la influencia está más en los padres de nosotros. Pero en sí a nosotros como que ya no nos importa tanto la religión..." (mujer, 18 años, universidad).

Estos relatos evidencian tensiones entre jóvenes frente a un "orden adultocéntrico" (Duarte, 2000), representado por efigies de autoridad como la Iglesia y el Estado, en el que se denotan jerarquías, bajo las cuales éstos ocuparían las categorías más bajas, en un lugar de difícil negociación. Sin embargo, sus discursos visibilizan apertura al trabajo con estos imperativos normativos desde una posición crítica.

(...) yo no soy religiosa y yo digo que una Biblia no me va a decir lo que tengo que hacer, porque yo creo en mis creencias, no soy atea, soy agnóstica, pero estoy en contra 
de la Iglesia y de la Biblia (..) si una Biblia dice que un género femenino y un género masculino se quieren juntar y formar la familia perfecta, yo digo que no, que así no es, entonces ¿̨por qué personas sienten atracción por el mismo género? (mujer, 15 años, colegio privado).

En este escenario, los discursos de los jóvenes entrevistados (principalmente en colegios privados y universidades) interpelan el rol de la Iglesia como productora de valores universales y sugieren una crisis de representación de sus dogmas. La Iglesia católica, principalmente, ha perdido credibilidad en tanto, cada vez más, se hace pública la humanidad de sus ministros y la inconsistencia de sus ordenanzas.

La Iglesia en verdad critica y critica, pero los curas son papás, son violadores, ellos mismos tienen hijos, entonces yo soy religioso, no soy católico porque yo creo a mi manera, no creo en la Iglesia porque encuentro que los curas son falsos y que ellos critican para ocultar todo lo malo que ellos tienen (hombre, 16 años, colegio privado).

Hay gente [de la Iglesia] que dice: "tú no tienes que hacer esto, tú no tienes que hacer esto otro" (...) y de repente veo y hay curas que se violan a niños chicos y monjas que están manoseando a niñas chicas y uno dice: ¿Dónde queda la moral? ¿Dónde queda la consecuencia? (mujer, 17 años, colegio privado).

De igual forma, los discursos de los jóvenes, principalmente en universitarios, identifican una relación entre moral religiosa y sus ideales y la producción de estereotipos que restringen la vivencia de la sexualidad, y junto con ello, un orden heteronormativo que reproduce y legitima inequidades entre hombres y mujeres. Asimismo, ante el reconocimiento de estas desigualdades, los sujetos elaboran en sus discursos nuevas normativas en torno a la sexualidad, apuntando a transformaciones de pautas de acción, otrora dominadas por instituciones como la Iglesia.

(...) yo creo que hay que cambiar a la Iglesia, porque se encarga de entregar la ética y la moral, y con la ética y la moral entra todo lo que son los tabús, los prejuicios (...) como el machismo ha disminuido junto con la caída de la Iglesia (hombre, 19 años, universidad).

(...) lo único que busca la Iglesia es negar los derechos de la mujer. Una vez (...) leí una cita, creo que de Juan Pablo II, que decía que dejar a una mujer como cura era mucho peor que la pedofilia y eso me enoja (hombre, 18 años, universitario).

\section{Estado}

De los discursos de los participantes emergió una segunda subcategoría en relación con instituciones normativas, el Estado y los aparatos gubernamenta- 
Jimena Silva-Segovia, Leyla Méndez-Caro y Jaime Barrientos-Delgado. Discursos sobre normas relativas a sexualidad en jóvenes del norte de Chile

les. Éstos aparecen como entidades que ejercen control en distintos ámbitos de la vida. Ambos coartan espacios de acción de la ciudadanía respecto a su sexualidad. Sin embargo, los jóvenes desdibujan la premisa de su configuración como meros efectos del control normativo y discursivo, y se vinculan con estas normativas, demandando ampliación de una ciudadanía sexual.

(...) en el Estado igual hay gente influyente, la mayoría esa influencia la ocupan negativamente (...) van enterrando esas ideas [de cambio desde agrupaciones informales y movimientos ciudadanos] y al final volvemos a lo mismo, a no tener ningún cambio ni a valorar tampoco a la gente que quiere cambiar la mentalidad (mujer, 17 años, universidad).

(...) El Estado es el que decide las cosas que debe enseñar, y educar a la población, y no solamente sobre el aborto sino que sobre muchos temas, la droga, la pena de muerte (...) educarla y después... hacerlo a través de los medios, la radio, la tele, mostrar informes, programas, debates, cosa que la gente se vaya creando una postura y una conciencia y ahí, fuera de ideales religiosos, llamar a un plebiscito y que sea la misma población la que decida qué es legal o no (hombre, 18 años, universidad).

Asimismo, en los relatos se emplaza al Estado a tomar un rol activo en el diseño y planificación de políticas en materia de educación sexual y derechos reproductivos. También se subraya la importancia de la injerencia de la población en la toma de decisiones. Los relatos advierten sobre la débil incorporación de la misma ciudadanía y voces juveniles en la construcción de políticas públicas y programas en sexualidad. Se devela una exigencia al ejercicio de una ciudadanía más activa.

Estas exigencias, de acuerdo al discurso de los jóvenes, se forjan en un contexto de estigmatización y discriminación presente en el intercambio entre ellos e instituciones gubernamentales. Específicamente, en el ámbito de los Derechos Sexuales y Reproductivos (DDSSRR) critican la actuación de los servicios de salud, en tanto éstos se posicionan simbólicamente desde el control, el orden y el castigo, construyendo una barrera entre la institución y los mundos juveniles.

(...) En los consultorios, creo que no debería hacerse tanto la discriminación, como mirar mal si va un joven, porque eso frena a la persona a ir a buscar un condón o ir a buscar pastillas. Creo que obviamente educar a los jóvenes, pero a la gente que trabaja con jóvenes (...) igual educarlos, es como no hacer diferencia. Por ejemplo, yo tengo casos de amigas que han creído estar embarazadas y no quieren ir porque les da vergüenza y tengo que ir yo, o sea yo he ido muchas veces y te miran como: "pobrecita, estai' embarazá" (...) (mujer, 17 años, universidad).

Asimismo, en la cita anterior se critica la verticalidad producida en las relaciones que infantilizan a los jóvenes depreciando su calidad de sujetos de 
derecho, a la vez que se enmascara en la protección y cuidado de éstos, dificultando procesos de cambio social.

En este contexto, también se reconoce en los relatos de los participantes a otros grupos sociales en desventaja frente a las legislaciones y acciones de aparatos de Estado. Este es el caso de las mujeres, quienes, en algunos discursos, son posicionadas como un sujeto de moral débil al momento en que éstas deben decidir sobre su sexualidad y autocuidado, siendo necesario el disciplinamiento institucional.

(...) el aborto debería ser ilegal debido a la irresponsabilidad de las niñas adolescentes, a excepción de las niñas abusadas que queden embarazadas. No creo que sea la mejor manera que una persona aborte (mujer, 16 años, colegio privado).

Como se advierte en la cita anterior, para un grupo de jóvenes es necesario que el Estado regule los efectos detonados por la desobediencia a la norma hegemónica, de la relación sexual como acto privativo para la reproducción. Asimismo, refuerzan la normativa de protección de los cuerpos de las mujeres como un receptáculo de vida que debe ser resguardado.

No obstante lo anterior, se advierten otros discursos, principalmente de jóvenes universitarios, quienes cristalizan demandas a favor de la despenalización del aborto, pues lo reconocen como un derecho legítimo de las mujeres, el cual devolvería autonomía y salud sobre sus propios cuerpos, tal como se reconoce en las siguientes citas.

(...) es mi cuerpo, yo decido si quiero o no lo quiero tener, (...) muchas personas opinan que no (...) resulta que yo voy a ser la que va a estar nueve meses con la guata (...) y si yo quiero abortarlo, bueno, es mi cuerpo, es mi decisión (...) igual yo lo encuentro más difícil porque el país ¿qué dice?: "es que no estamos preparados" (mujer, 19 años, universidad).

(...) yo sí estoy de acuerdo con el aborto (...) lo veo por mí, porque de verdad yo no me imagino nueve meses con una guata, no imagino teniendo un niño, teniendo que postergar mis estudios, mi vida, mis amistades, todo por tener un hijo (...) (mujer, 18 años, universidad).

Por otro lado, los discursos de los jóvenes evidencian dificultades para llevar a cabo procesos de cambio, éstos mediados por las reparticiones de gobierno y la vinculación entre Iglesia y Estado. En el primer caso, argumentan que el funcionamiento del gobierno actual antepone sus ideales "conservadores" al momento de gobernar. Estas limitaciones de orden ideológico se convierten en imágenes inquebrantables - tal como se advierte en la siguiente cita-, que promueven indefensión frente a los procesos de cambio. 
Jimena Silva-Segovia, Leyla Méndez-Caro y Jaime Barrientos-Delgado. Discursos sobre normas relativas a sexualidad en jóvenes del norte de Chile

[Sobre aborto] ¿Con el gobierno actual? No, yo pienso que no, ni jodiendo, va a ser re difícil, si es derecha y son súper conservadores, yo creo que van a hacer lo imposible, como lo están haciendo con el matrimonio homosexual para que no pase (mujer, 19 años, universidad).

En el siguiente caso, los discursos realzan el dispositivo Iglesia-Estado, el cual sigue operando sin secularizarse en la práctica. De acuerdo con esto, los discursos de un grupo de jóvenes, en su mayoría hombres de colegios privados, defienden la idea de un Estado autónomo frente a la moral religiosa, idealizando su funcionamiento.

Yo creo que la Iglesia se aprovecha del poder que tiene en hartos sentidos (...) lo que debería mandar es la ley y listo, no tiene nada que ver la Iglesia, para eso existen las leyes (...) Yo estoy a favor [matrimonio entre personas del mismo sexo], porque yo creo que es una cosa de libertad, una cosa de elegir. Si a mí me gustaran los hombres y yo pudiera forjar un matrimonio gay y tener un hijo, al final es lo que uno quiera hacer. Eso ya debería tomarse como algo normal (hombre, 16 años, colegio privado).

Igual yo creo que por el niño, ahora que en Chile se apruebe el matrimonio gay (...) porque a los niños los discriminaría todo el mundo, pero si aprobaran la ley y pasaran 10, 5, 6 años ya estaría más abierta la mentalidad hacia ese tema y ahí podría abrirse una pequeña posibilidad de que tengan hijos (hombre 2, 16 años, colegio privado).

De esta forma, si bien se cuestionan los mandatos y normativas religiosas, se desplaza la adhesión a otros modelos institucionales - en un discurso no plenamente consciente- como el modelo heteronormativo de la familia y una visión romántica de las leyes, las cuales son situadas como entidad primaria para la transformación de dichos procesos, en detrimento del agenciamiento personal frente a este debate.

Por otro lado, emergieron discursos de universitarios, que con menos idealización hacia el Estado y sus leyes, sugieren que mientras intervenga la moral religiosa en la toma de decisiones, se seguirá fortaleciendo inequidades a la hora de legislar. Este dispositivo repercute en la sexualidad de la ciudadanía, pues antepone su corpus de ideales, avasallando la diferencia.

\section{Escuela}

Sobre la base del control que ejercen las instituciones educativas en sexualidad, y la implementación/no implementación de programas en educación sexual, los discursos biomédicos de carácter instituyente regularían la sexualidad juvenil, a través del diseño curricular de la educación sexual. En algunos establecimientos se trabajaría, por ejemplo, naturalizando el género median- 
te argumentaciones desde la medicina clásica, razonamientos que legitiman la forma tradicional de ser hombre o ser mujer, en función de un dispositivo de control heteronormativo. Esto lo podemos advertir en la siguiente cita.

(...) mi profesor de Biología nos explicó lo siguiente y yo concuerdo mucho con él; para los hombres la sexualidad, su placer sexual (...) son completamente distintas que el de las mujeres, [pues ellos] se excitan sexualmente muy fácilmente (...) También pueden fingir querer a alguien con tal de satisfacerse sexualmente, en cambio las mujeres son más de sentimientos, si se excitan no es con cualquier hombre, siempre es con alguien específico (...) (mujer, 16 años, colegio particular).

La entrevistada naturaliza una concepción heteronormativa otorgada por un referente académico, emergiendo como un sujeto obediente a la norma. Asimismo, adhiere sin cuestionamientos al discurso del profesor que ostenta estatus científico y, por tanto, verosímil, construyendo y reproduciendo las concepciones tradicionales e inequitativas de los binomios femenino y masculino. Esta misma lógica se desenvuelve en los discursos sobre sexualidad, presentes en la siguiente cita. Sin embargo, en ésta se ven reflejados otros grupos de jóvenes, quienes interpelan el contenido de las clases en tanto mecanismos de control de prácticas sexuales, develando una tensión entre la precarización de los contenidos de educación sexual -centrados en la enfermedad y el malestar de las juventudes frente a este tipo de educación sexualy la necesidad de reestructuración de ésta.

Las clases de educación sexual a mí me causaban mucha gracia porque lo que hacían no era educar sobre las prácticas sexuales, sino que era meter miedo para que en el fondo nadie tuviera sexo y pensáramos que el sexo es lo más horrible y que por tener un coito te iba a dar síflis, gonorrea (...) o quedarías embarazada (...) y nadie te explicaba lo que era el acto sexual ni los métodos ni nada (mujer, 20 años, universidad).

Así, el discurso biomédico mediante el uso de la "educación del miedo", trataría de moldear los significados de las sexualidades de los jóvenes, separando afectividad y sexualidad, y coartando la curiosidad, deseos y placeres frente a la inminente transmisión de una enfermedad o embarazo no planificado.

Una vez que estas últimas situaciones se concretan, como el embarazo en jóvenes, el desplazamiento ya no sólo actúa a nivel simbólico, sino que se materializa en la restricción de espacios para las mujeres, quienes de acuerdo con los discursos de los participantes, son quienes se ven mayoritariamente perjudicadas al momento de la maternidad, pues a pesar de existir un marco legal que ampara a las mujeres jóvenes embarazadas en sus trayectorias educativas, seguiría imperando sin mayores cambios una estructura social que produce 
Jimena Silva-Segovia, Leyla Méndez-Caro y Jaime Barrientos-Delgado. Discursos sobre normas relativas a sexualidad en jóvenes del norte de Chile

y reproduce desigualdades para este grupo, sobre la base del mantenimiento de un orden patriarcal.

(...) Pero aunque se hagan cargo igual uno [como mujer] igual se lleva la mayor parte, por ejemplo, ellos pueden seguir estudiando, pero si una no tiene el apoyo de alguien, de los papás el $100 \%$, tampoco puede hacerlo y uno está obligada a quedarse con el bebé (...) (mujer 2, 16 años, colegio privado).

Yo igual [me haría cargo del embarazo], yo creo que eso es un miedo que le meten más a las mujeres (...) Si dejas a una mina embarazada a esta edad, mal para los dos (...) (hombre, 16 años, colegio privado).

El segundo relato representa los discursos de varios hombres jóvenes de colegios privados y universidades, quienes reconocen la influencia sociocultural y de género en las relaciones entre hombres y mujeres al momento de un embarazo, evidenciando una reflexión crítica sobre mandatos socioculturales que relegan tanto a hombres como a mujeres a reproducir modelos de género inequitativos.

Finalmente, si bien en materia de educación sexual se advirtieron diferencias entre colegios, éstas no apuntarían a la distribución público/privado, sino a la distinción entre quienes no imparten educación sexual, quienes sí lo hacen y los lineamientos que rigen el desarrollo de éstos (biomédico conservador/integral). No obstante, también de acuerdo con los relatos, habría una conexión entre precarización de programas de educación sexual y establecimientos educacionales municipales.

[Educación sexual] son ramos estables en primero y segundo me hacían sexualidad y vida (...) En tercero, ya me lo quitaron (...) los preservativos, la forma de cuidarse, la forma de estar con un hombre, la forma de estar con una mujer (...) Los encontraba interesante porque en esas clases yo siempre me sentaba de las segundas o de las terceras (...) La vida sexual, que dos parejas sean del mismo sexo, o de la vida que uno podría llevar si no se cuida (...) Si porque uno aprende más (mujer, 16 años, colegio municipal).

(...) en el [colegio] de nosotros, está impartiendo la educación sexual en tercero medio

(...) y más de la mitad de mis compañeros ya habían iniciado las relaciones mucho antes, entonces, de qué estamos hablando (hombre, 17 años, colegio particular).

De esta forma, los relatos nos sugieren una educación sexual descontextualizada frente a las realidades de los jóvenes y precarización de instancias de formación y discusión al interior del aula. En algunos casos, se intenta entregar educación sexual integral, como en el primer relato. Sin embargo, luego la educación sexual se elimina del currículo.

En la segunda cita si bien se incorpora como materia, se da de forma tardía, y en el tercer relato se alude a la superficialidad en el abordaje de la sexua- 
lidad y afectividad. En todos los casos se refleja la carencia de programas de educación sexual y afectividad, y de profesionales especializados y dedicados a la potenciación de esta área.

\section{Conclusión}

Ante las preguntas: ¿cómo operan los discursos valóricos provenientes de instituciones como la Iglesia, el Estado o la escuela sobre la sexualidad juvenil de estudiantes antofagastinos/as? y ¿cómo estos/as jóvenes se relacionan con los discursos institucionales que recaen sobre el cuidado de sus cuerpos?, hemos analizado e interpretado discursos juveniles, observando que estas tres instituciones intervienen en la estructuración de la sexualidad juvenil a través de distintas esferas educativas (por ejemplo, pública, privada, universitaria; también a través de padres, madres, profesores y representantes de la Iglesia).

Sin embargo, también se advirtió el desamarre y debilitamiento de la representación que poseen sobre la juventud, aspecto ya señalado en el planteamiento teórico. Así, cobró fuerza en la mayoría de los discursos juveniles una reflexión crítica hacia estas entidades.

En el caso de la Iglesia, ésta se configuró en los discursos como médium simbólico de comunicación e instrumento de conocimiento (Bourdieu, 2009), a través del cual los jóvenes interpretaron sus vivencias afectivo-sexuales. En los discursos juveniles, principalmente de aquellos provenientes de colegios privados católicos, se observa la exacerbación de mandatos pro-vida, discursos sexistas y homófobos. No obstante, también se observó una crisis de representación de la Iglesia, visibilizada en discursos de jóvenes universitarios y de un grupo pequeño de colegios privados.

De acuerdo con ésta, se interpela a la Iglesia como dispositivo de control de la sexualidad de los jóvenes, sobre todo de las mujeres, y de la regularización de los cuerpos y sus prácticas, propiciando el mantenimiento de un orden político inequitativo en esta materia.

En relación con el Estado develamos demandas por parte de estudiantes universitarios y aquellos de colegios que apuntaron a la formulación de un programa de educación sexual integral, considerando principalmente las voces juveniles al momento de levantar sus lineamientos de acción, en un inminente ejercicio de construcción de una ciudadanía sexual. Ante este escenario, aparece una construcción de la juventud basada en la validación de un enfoque evolutivo. 
Desde esta perspectiva, la moratoria se configura como paradigma hegemónico (Borzese et al., 2004), encarnando y legitimando históricamente las políticas de Estado, que devienen en la infantilización del sujeto joven. Asimismo, como refiere Dides (2007), el control social estatal es sublimado por parte de instituciones jurídicas, morales y bioéticas que se encargan de moldear las subjetividades juveniles, manteniendo la vigilancia implícita de la sexualidad a lo largo de sus trayectorias vitales.

En cuanto a la tercera institución, la Escuela, advertimos que ésta se configura en el espacio físico, donde la Iglesia y el Estado coaccionan sobre los cuerpos juveniles (Foucault, 2002). Es en este campo donde a través de métodos sutiles de persuasión y disciplinamiento se educa a los cuerpos en materia de sexualidad y afectividad, y se configuran los habitus de socialización de género como "proceso mediante el cual el orden social se inscribe progresivamente en las mentes (...) y se encarna en nuestro cuerpo en forma de hexis corporal" (Rodríguez, 2003: 93).

En los relatos juveniles identificamos demandas hacia la implementación de programas de educación sexual, los cuales, como ya señalamos, se encuentran precarizados y a merced de las políticas de educación sexual de los establecimientos. Estas políticas, en la mayoría de los casos analizados, se centran en el abordaje de la sexualidad desde un enfoque biomédico clásico, el cual perpetúa y legitima mandatos de género y activa dispositivos heteronormativos, en un contexto que limita la emergencia del autocuidado y prevención de las prácticas sexuales reales - de acuerdo con contextos específicos- de las juventudes.

Cabe señalar que esta discusión respecto a la precarización de programas de educación sexual y a la percepción negativa de la intervención institucional en materia de sexualidad y afectividad juvenil, es desarrollada principalmente en dos grupos de jóvenes pertenecientes a colegios privados y universidades.

Por su parte, en jóvenes de establecimientos municipales, se evidencia una baja producción de reflexiones críticas e interpelación a dichas instituciones, advirtiéndonos que el lugar donde se estudia incide en el acceso a capitales culturales, la producción intelectual y circulación de discursos disidentes (Bourdieu, 2008), que en este caso denotan la precarización y marginación de colegios públicos, frente a privados y universidades.

Este escenario de brechas educacionales se exacerba en Chile desde la municipalización de la enseñanza en 1986, bajo la cual los municipios y no el Estado se hacen cargo de la administración de ésta y desde allí cada año observamos, por ejemplo, diferencias significativas entre los resultados de 
SIMCE (Sistema de Medición de la Calidad de la Educación) entre colegios públicos y privados; y al mismo tiempo, vemos bajo rendimiento de estudiantes de colegios públicos en la Prueba de Selección Universitaria (PSU).

De acuerdo con los párrafos anteriores, pudimos dar cuenta de la configuración de sujeto joven construida en torno a la sexualidad y autocuidado en tanto vinculación con las normativas institucionales. Respecto a esto, observamos realidades fuertemente estructuradas; sin embargo, al mismo tiempo fue interesante advertir en sus discursos espacios que permiten incertidumbre e imprecisión (Rodríguez, 2003) en función de una potencial ciudadanía sexual.

Frente a lo anterior, si bien a nivel contextual atravesamos por un modelo de sociedad, en el cual es difícil establecer normas morales claras que se correspondan con principios éticos determinados, en una denominada "crisis normativa” (Marín, 2008), es necesario no solamente situar la discusión en una relación causal entre norma inscrita y conducta, sino más bien relevar la elaboración moral del sujeto.

Como diría Araujo (2009a), al momento de pensar en sujetos y normas, debemos centrarnos en su relación permanente y dinámica basada en ideales, experiencia social y configuración de sujeto. Por lo tanto, es necesario reconocer a los sujetos jóvenes como entes activos alejados de una esfera de indefensión pasiva o de acatamiento, dado que los ideales y normas en una sociedad son volubles, siempre a merced de los vaivenes de la experiencia social. A su vez, esto propicia espacios para el cambio, tal como se advirtió en las narraciones de los jóvenes entrevistados. Todo lo anterior, es sobre la base de una configuración de sujeto siempre inconclusa, resultado de un trabajo de conformación permanente en el marco de expectativas, restricciones y posibilidades sociales.

Finalmente, en relación con las limitaciones del estudio, se sitúa el débil análisis de otras instituciones relevantes como la familia en el contexto minero, necesaria de profundizar en futuros trabajos de investigación, de acuerdo al lugar que ocupa el sujeto en torno a la norma en un contexto de homosociabilidad minera donde "la identidad masculina hegemónica se intensifica (...) y se refuerzan los estereotipos de género asociados tanto a hombres como a mujeres" (Salinas y Barrientos, 2011: 436).

Por otro lado, se sugiere ampliar los criterios de inclusión de los participantes, dado que sólo se trabajó con jóvenes de enseñanza media en educación formal. De esta forma, futuros trabajos deberían considerar a jóvenes no escolarizados, pues sus intercambios sociales y afectivos-sexuales se desen- 
vuelven alejados de la institución escuela, siendo interesante explorar aquellos campos discursivos construidos fuera de la socialización de esta entidad. Asimismo, es importante ampliar los tramos de edades, recuperando relatos de jóvenes de enseñanza básica, dada la precoz entrada de los jóvenes en la vida sexual y afectiva (INJUV, 2009), y además, considerar otros discursos de representantes de instituciones, como familiares, profesores y empleados estatales.

También se advierte como limitación el tipo de técnica utilizada. Se requiere, además de una entrada individual, un abordaje grupal complementado con metodologías innovadoras. En la recolección de información, se observaron dificultades en los jóvenes para identificar y verbalizar experiencias sobre la vida sexual y afectiva.

Por ello se sugiere ocupar herramientas que faciliten la emergencia discursiva y descripción de las vivencias, por ejemplo, mediante abordajes etnográficos, grupales y técnicas de mapas corporales (Silva, 2009); a la vez, fortalecer las capacidades técnicas de los investigadores para el uso de éstas e interpretación de sus hallazgos. Este acercamiento acortaría las brechas entre discursos y prácticas juveniles y nos ayudaría a poner en situación a los sujetos, para aproximarnos a las prácticas y vivencias cotidianas.

Asimismo, se sugiere realizar un análisis de "tipos" de sujetos y su relación con las normas. Por supuesto, no con el afán de esencializar configuraciones de sujeto, sino como aporte para el estudio de las normas, sexualidad y autocuidado y focalización de lineamientos de intervención, toda vez que se reconozca lo inacabado de su conformación, sobre la base de focalización de estrategias de investigación acción.

Por último, es necesario considerar un análisis generacional de las caracterizaciones de sujeto-joven, de acuerdo al cual se reflexione sobre cómo los jóvenes de estas generaciones se relacionan con la norma - a propósito de los cambios globales, sociales y culturales-, pues no podemos observar a este grupo fuera de su inscripción cultural, histórica y social, la cual nos advierte de una "vida sexual y afectiva cada vez menos regulada por instituciones como la Iglesia, la educación o el Estado y, cada vez más, [regulada] por los sujetos de manera situacional y contingente" (Barrientos y Silva, 2006: 130).

\section{Bibliografía}

Alonso, Luis (2003), La mirada cualitativa en sociología, Madrid: Fundamentos.

Araujo, Kathya (2009a), "Estado, sujeto y sexualidad en el Chile postdictatorial”, en Revista Nomadias, núm. 9, Chile: Universidad de Chile. 
Araujo, Kathya (2009b), “El ordinario trabajo moral del sujeto”, en Kathya Araujo, ¿Se acata pero no se cumple? Estudios sobre las normas en América latina, Santiago: Lom ediciones.

Barrientos, Jaime y Jimena Silva (2006), De la restricción hacia la equidad, Antofagasta: Ediciones Universidad Católica del Norte.

Borda, Orlando (1981), Ciencia propia y colonialismo intelectual, Bogotá: Carlos Valencia Editores.

Borzese, Dana, López Cecilia y Ruiz Roberta (2004), “Visiones sobre la juventud en Argentina: de los problemas a los derechos”, en Dávila, Óscar [ed.], Ser joven en Sudamérica, diálogos para la construcción de la democracia regional, Chile: Centro de Estudios Sociales (CIDPA).

Bourdieu, Pierre (2008), Capital cultural, escuela y espacio social, Buenos Aires: Siglo XXI Editores.

Bourdieu, Pierre (2009), La eficacia simbólica. Religión y politica, Buenos Aires: Biblos.

Bozon, Michel (2004), La nueva normatividad en sexualidad. Construyendo una agenda temática en sexualidad, Antofagasta: Ediciones Universidad Católica del Norte.

Dides, Claudia (2007), Chile panorama de sexualidad y derechos humanos, Chile: Centro Latinoamericano de Sexualidad y Derechos Humanos.

De Villers, Guy (1999), "La historia de vida como método clínico", en Revista Proposiciones, núm. 23, Chile: Universidad de Chile.

Foucault, Michel (2002), Vigilar y castigar: nacimiento de la prisión, Buenos Aires: Siglo XXI Editores.

Gallegos, Francisca, Leyton, Daniel y Leyla Méndez (2009), Estudio de diagnóstico y caracterización de las y los jóvenes de la región de Antofagasta, Chile: Instituto Nacional de la Juventud.

Grau, Olga [comp.] (1997), Familia: un grito de fin de siglo. Discurso, género y poder, Santiago: LOM ediciones.

Guzmán, Virginia y Lorena Godoy (2009), “Individuación y normatividad de género: la construcción de proyectos biográficos”, en Araujo, Kathya [ed.], ¿Se acata pero no se cumple? Estudios sobre normas en América Latina, Santiago: LOM ediciones.

Htun, Mala (2010), Sexo y Estado. Aborto, divorcio y familia bajo dictaduras y democracias en América Latina, Santiago: Ediciones Universidad Diego Portales.

Instituto Nacional de la Juventud (INJUv) (2009), Sexta Encuesta Nacional de la Juventud, Chile: Informe Gubernamental.

Novaes, Regina y Mauricio Santoro (2008), “Ser joven na América do Sul: Um epílogo”, en Dávila, Óscar [ed.], Ser jóvenes en Sudamérica, diálogos para la construcción de democracia regional, Chile: Centro de Estudios Sociales (CIDPA).

Olavarría, José (2009), "Las ¿nuevas? paternidades. La organización del trabajo, la familia y la globalización", en Lerner Susana y Lucía Melgar [eds.], Familias en el siglo XXI: realidades diversas y politicas públicas, México: UNAM.

Palma, Irma (2007), “Trayectorias sexuales, vínculos y contextos en la generación que hace su entrada en la sexualidad activa en democracia”, en Revista Observatorio de Juventud, año 3, núm. 9, Chile: Instituto Nacional de la Juventud. 
Jimena Silva-Segovia, Leyla Méndez-Caro $y$ Jaime Barrientos-Delgado. Discursos sobre normas relativas a sexualidad en jóvenes del norte de Chile

Portocarrero, Gonzalo (2009), "Moralismo, contestación y cinismo como posiciones de enunciación de los juicios morales en la juventud peruana”, en Araujo, Kathia [ed.], ¿Se acata pero no se cumple? Estudios sobre normas en América Latina, Santiago: LOM ediciones.

Rodríguez, María del Carmen (2003), La configuración del género en los procesos de socialización, Oviedo: Ediciones KRK.

Silva, J. (2009), Relaciones de poder entre mujeres chilenas de dos generaciones. Alianzas, transgresiones, disidencias, oposiciones entremadres ehijas. Tesis doctoral en Antropología Sociocultural, Chile: Facultad de Ciencias Sociales y Jurídicas, Universidad Católica del Norte/ Universidad de Tarapacá.

Urriola, Ivonne (2008), “El género: ¿ruptura del consenso político en Chile?, 1989-2000”, en Revista de Historia Actual, núm. 16, España: Asociación de Historia Actual.

Van Dijk, Teun (2008), Ideología y discursos, Barcelona: Ariel.

Vera, Antonieta (2005), "Chilenos que Importan: Familia y pensamiento conservador en el Chile actual”, en Wilson, Angélica [ed.], Conservadurismo y trasgresión en Chile: Reflexiones sobre el mundo privado, Santiago: Lom ediciones.

\section{Recursos electrónicos}

Barrientos, Jaime (2006), “¿Nueva normatividad del comportamiento sexual juvenil en Chile?”, en Revista Última Década, núm. 24, Chile: Centro de Estudios Sociales (CIDPA). Disponible en: http://www.redalyc.org/articulo.oa?id=19502405 [3 de marzo de 2012].

Duarte, Claudio (2000), “ ¿Juventud o juventudes? Acerca de cómo mirar y remirar a las juventudes de nuestro continente”, en Revista Última Década, núm. 13, Chile: Centro de Estudios Sociales (CIDPA). Disponible en: http://www.scielo.cl/scielo. php?script=sci_arttext\&pid=S0718-22362000000200004 [18 de junio de 2012].

Marín, Tomás (2008), “Nuevas formas de moralidad en los y las jóvenes chilenos”, en Revista Última Década, núm. 28, Chile: Centro de Estudios Sociales (CIDPA). Disponible en: http://www.scielo.cl/scielo.php?script=sci_arttext\&pid=S0718-2236200800010 0008 [23 de agosto de 2012].

Méndez, Leyla y Manuel Cárdenas (2012), "Hacia la construcción de un modelo comprensivo de análisis de la 'situación de inmigración' de mujeres sudamericanas en Chile”, en Revista Psicoperspectivas, núm. 1, Chile: Pontificia Universidad Católica de Valparaíso. Disponible en:

http://www.scielo.cl/scielo.php?pid=S0718-69242012000100012\&script=sci_arttext [11 de marzo de 2012].

Palma, Irma (2010), “Trayectorias sexuales, preventivas y sociales en el embarazo no previsto de los segmentos juveniles en Chile, en Revista Última Década, núm. 33, Chile: Centro de Estudios Sociales (CIDPA). Disponible en: http://www.scielo.cl/pdf/udecada/ v18n33/art06.pdf [3 de marzo de 2012].

Ruiz, Soledad et al. (2011), "Jóvenes secundarios de hoy: estudio sobre su visión de la sociedad desde los conceptos de anomia y alienación psicosocial”, en Revista Última 
Década, núm. 35, Chile: Centro de Estudios Sociales (cIDPA). Disponible en: http:// www.scielo.cl/scielo.php?pid=S0718-22362011000200009\&script=sci_arttext [8 de septiembre de 2012].

Salinas, Paulina y Jaime Barrientos (2011), "Los discursos de las garzonas en las salas de cerveza del norte de Chile. Género y discriminación”, en Revista Polis, núm. 29, Chile: Universidad Bolivariana de Chile. Disponible en: http://www.scielo.cl/pdf/polis/ v10n29/art19.pdf [9 de julio de 2012].

Sharim, Dariela (2005), "La identidad de género en tiempos de cambio: Una aproximación desde los relatos de vida”, en Revista Psykhe, núm. 29, Chile: Pontificia Universidad Católica de Chile. Disponible en: http://www.scielo.cl/scielo.php?pid=s071822282005000200002\&script=sci_arttext [24 de mayo de 2012].

Vera, Antonieta (2009), "Una crítica feminista a la madre pública postdictatorial: los discursos de género en la campaña presidencial de Michelle Bachelet", en Revista Nomadias, núm. 10, Chile: Universidad de Chile. Disponible en: http://www.revistas. uchile.cl/index.php/NO/article/view/15133 [3 de abril de 2012].

Jimena Silva Segovia. Doctora en Antropología, docente investigadora de la Universidad Católica del Norte (UCN), Antofagasta, Chile. Líneas de investigación: género, sexualidad y juventudes. Publicaciones recientes: Jaime Barrientos y Jimena Silva, "Sexual initiation of young people in Chile: a qualitative study”, en International Journal of Sexual Health (2013); Jimena Silva, Jaime Barrientos y Ricardo Espinoza-Tapia, "Un modelo metodológico para el estudio del cuerpo en investigaciones biográficas: los mapas corporales", en Revista Alpha (2013); Jimena Silva y Marcelo Lufín, "Approaches to the Afro-Colombian Experience in Chile South-South Immigration Toward the Northern Regions", en Journal of Black Studies, Sage Jorunal (2013).

Leyla Méndez Caro. Magister en Psicología Social, académica e investigadora en la Universidad de Antofagasta Angamos, Chile. Líneas de investigación: género, sexualidad, juventudes, inmigración. Publicaciones recientes: Leyla Méndez Caro, Manuel Cárdenas, Fabiola Gómez, Susan Yáñez, "Situación de inmigración de mujeres sudamericanas en Chile: hacia un modelo comprensivo" (artículo de resultados), en Psicologia \& sociedade, vol. 24, núm. 3, ABRApso (Brazilian Society of Social Psychology) (2012); Leyla Méndez Caro y Manuel Cárdenas, "Hacia la construcción de un modelo comprensivo de análisis de la 'situación de inmigración' de mujeres sudamericanas en Chile” (artículo metodológico), en Revista Psicoperspectivas, vol, 11, núm. 1 (2012); Leyla Méndez Caro, Jimena Silva, Beatriz Pereira y Eileen Flores, "Discursos inscritos sobre el cuerpo: estudio sobre las implicancias de la mastectomía en mujeres chilenas”, en Salud \& Sociedad, vol. 3, núm. 2, 
Antofagasta, Chile (2012).

Jaime Barrientos Delgado. Doctor en Psicología Social, docente investigador de la Universidad Católica del Norte (UCN), Antofagasta, Chile. Líneas de investigación: género y sexualidad. Publicaciones recientes: Jaime Barrientos y Jimena Silva, "Sexual initiation of young people in Chile: a qualitative study”, en International Journal of Sexual Health (2013); Jimena Silva, Jaime Barrientos, Ricardo Espinoza-Tapia, "Un modelo metodológico para el estudio del cuerpo en investigaciones biográficas: los mapas corporales”, en Revista Alpha (2013); Jaime Barrientos, Manuel Cárdenas, Fabiola Gómez y Dolores Frías-Navarro, "Assessing the Dimensionality of Beliefs about Children's Adjustment in Same-Sex Families Scale (BCASSF) in Chile", en Sexuality and Social Policy (2013).

Recepción: 29 de noviembre de 2012.

Aprobación: 31 de julio de 2014. 
